\title{
Is Darwinism a thermodynamic necessity?
}

The concept of fitness has helped evolutionists avoid the accusation of tautology, but there is a case for asking that the notion should be defined in operational terms as well as by its consequences.

THE argument that the theory of natural selection is nothing but a tautology, due in its most elegant form to Sir Karl Popper, now hardly ever keeps evolutionists or population geneticists awake at night. That, no doubt, is how it should be. If artificial selection can be used to breed specialized forms of animals or plants, why should not natural selection, by the slings and arrows of the changing environment, yield species welladapted to the world in which they find themselves?

That is a defensible position, but it does not remove the nagging doubt. Most simply, the tautology is the observation that if, in the "survival of the fittest", the fittest are identified with the survivors, the principle of natural selection amounts to the empty slogan "survival of the survivors". This proposition does not explain why there should be survivors anyway.

Evolutionists seek to avoid the difficulty by the intermediate concept of "fitness", a measure of the adaptation of an organism to the present environment that Darwin would have related to the numbers of living offspring it is likely to leave behind. There are many circumstances in which the notion works well and simply as "for example" in accounting for the survival of the sickle-cell gene in regions where malaria was once endemic; the relative immunity of heterozygotes to infection (compared with those carrying normal genes) outweighs the decrement of fitness of those carrying two copies of the gene, who are likely to suffer from congenital and often-fatal anaemia.

Latterly, fitness has been more generally defined as the likelihood that an organism will contribute genes to the future gene-pool, thus allowing for the evolution of behaviour patterns anthropomorphically called altruistic (and for the notion of the "selfish gene"), but the principle is the same. The trouble, however fitness is defined, is that it is a post hoc measure of the course of evolution; to make a theory of evolution, one has to enumerate all possible genetic changes, estimate the degree to which they are likely to be represented in the future gene-pool and only then to tell in what way a population is evolving. Is there not some way in which fitness in this context might be more directly defined by the degree to which the metabolic processes on which an organism depends for its survival are biochemically matched, or adapted, to the environment?

If there were such a generally applicable definition, it would be possible to prove that evolution is an unavoidable necessity or, more accurately, to identify the environmental parameters (and their rates of change with time) conducive to evolution. One source of confusion, but occasionally of conceptual difficulty as well, is that natural selection works on individual organisms, but that what evolves is a species or even an aggregation of species.

A further difficulty is that there is an unbridged gap between the mechanisms, necessarily biochemical at root, by which an organism can be counted well-adapted to its environment and the likely selection of its genes into the next generation's gene-pool. And as things are, there are not the physical criteria by which it can be judged whether the chance of selection is maximized.

This is the position from which J.-L. Torres, a Mexican from the University of Michoacan working at the International Centre for Theoretical Physics at Trieste, embarks on an attempt to apply the rules of nonequilibrium thermodynamics to the calculation of fitness in the mechanistic sense (Il Nuovo Cimento 13D, 177; 1991). His objective is to find some way of measuring an individual's fitness by the degree to which its chief metabolic processes satisfy several thermodynamic criteria.

The working criteria are straightforward enough. A high degree of fitness would imply that metabolic processes are maximally efficient and, moreover, that they are maximally productive of whatever products the organism requires of them. On the other hand, the rate of entropy production should be a minimum, as should be the rate at which energy resources are consumed. In some circumstances, other criteria - for example, the rate of biomass production - might have to be reckoned with. By listing these criteria, Torres pretends no more than that they are a collection of plausible candidates in the construction of some composite indicator of what might be called instantaneous fitness.

But does not this make a horrendously complicated problem? The task of applying criteria such as these, not necessarily compatible with each other, to all the major metabolic cycles of an organism is evidently not child's play. But Torres is merely concerned to argue that it may be a little simpler than it seems.

Using such scant experimental data as there are for the production of ATP by the conversion of glucose into water (in red blood cells) and by respiration (in animal cells), he persuades himself, for example, that the expectations of conventional equilibrium thermodynamics do not necessarily apply. For then one would expect there to be a sharp conflict between the rate of ATP production and the efficiency of the process; the faster, the less efficient. In the classical Carnot cycle, the most efficient heat engine there can be, all processes are taken to be infinitely slow so that the power is infinitesimally different from zero. Why should ATP production be different?

In reality, Torres argues, in the nonequilibrium steady-states that prevail when ATP is produced in living organisms, physiological conditions correspond to a situation in which the measured efficiency of the reaction (measured as the fraction of free energy converted) is greater than a half and the rate of conversion is a maximum or nearly so.

In principle, at least, this points to a solution of this part of the problem. If the rate of ATP production and the efficiency of the process are to be regarded simultaneously as measures of fitness in the sense of matching the performance of an organism and the external demands of its environment, there will be a trade-off to be struck whose consequences are not nearly as depressing as pageone thermodynamics would lead one to expect.

That is a cheerful signpost, but not nearly half the real battle of defining, let alone calculating, some kind of dynamical instantanous measure of fitness. Indeed, there is a what may be a little more than a procedural difficulty tucked away in an appendix in which Torres sets out to derive a general form for the velocity of a network of reactions catalysed by enzymes (which is a necessary step in calculating the flux of reactants and products in a steady-state). $\mathrm{He}$ starts from the elementary textbook statement of the law of mass action that the rate of an uncatalysed reaction is determined by the concentrations of the reactants each raised to an integral power equal to its stoichiometric coefficient, and then supposes that the effect of enzymes is to multiply each coefficient by a constant different from the number one.

Many biologists will be tempted to regard this as a pointless exercise, but they will be mistaken. What matters in, say, population genetics, may be the degree to which an organism, by the totality of its behaviour, contributes genes to the future gene-pool, but it is a laudable exercise to see whether that state of affairs is reflected in the way in which the metabolism of the organism is matched biochemically to the environment. And, for what it is worth, Torres admits that there are "nontrivial gaps in the argument", which is a seemly sign of modesty.

John Maddox 